\title{
Mechanical and Electrical Characterization of Carbon Nanofibers produced from Water Soluble Precursors
}

Lee D. Cremar*, Javier Acosta-Martinez, Alexsandra Villarreal, Alfonso Salinas, and Karen Lozano*

Mechanical Engineering Department, University of Texas-Rio Grande Valley, 1201 West University Drive, Edinburg, TX 78539, USA

\begin{abstract}
This study presents the thermo-physical, electrical, and mechanical characterization of fine carbon fibers produced from water-soluble polymer precursors and low temperature processes. These fibers were developed utilizing the Forcespinning ${ }^{\circledR}$ technology which utilizes centrifugal force to spin fibers. Polyvinyl alcohol was used as the precursor material, and fibers were developed and deposited in a non-woven configuration. The resultant nonwoven fiber mats were subjected to a dehydration process through exposure to sulfuric acid vapors. The partially carbonized mats were heat treated at $850^{\circ} \mathrm{C}$. The produced porous nonwoven carbon fiber based mats have micro-and mesoporosity with a final fiber average diameter of $191 \mathrm{~nm}$. The electrical volume resistivity of the polymeric nanofibers was $8 \times 10^{14}$ $\Omega \cdot \mathrm{cm}$ and it dropped to $165 \Omega \cdot \mathrm{cm}$ for the chemically treated carbon fiber mat and to 0.407 $\Omega \cdot \mathrm{cm}$ for the subsequently heat treated fibers. The electromagnetic (EMI) shielding effectiveness (SE) was observed to be 20 and $40 \mathrm{~dB}$ for the chemically treated fibers and heat treated fibers respectively. The tensile stress for the chemically treated fibers was $43 \mathrm{MPa}$ with a strain of $18 \%$ while the subsequently heat-treated fibers exhibited a stress of $124 \mathrm{MPa}$ and strain of $21 \%$.
\end{abstract}

\footnotetext{
*Corresponding author. Tel: 956 665-7020 Email: karen.lozano@utrgv.edu; lee.cremar@utrgv.edu
} 


\section{Introduction}

Carbon fibers have been a material of interest for several decades due to their chemical stability and mechanical and electrical properties. Researchers are actively searching for materials and methods to lower production costs and enhance properties. As is, it is estimated that the global carbon fiber market has grown to over 12,500 tons per year [1]. Carbon fibers are mostly used as structural reinforcement for the aerospace, automotive, and sports industries [2]. Recent studies are actively focusing on carbon based nanomaterials such as carbon nanotubes (CNTs), nanofibers, and graphene given its multifunctional properties that have shown promising applications in a variety of fields. These carbon based materials have shown high surface area and display enhanced electrical-thermal conductivity [2-4]. Studies have shown potential use of carbon nanomaterials as electrodes (energy conversion and storage applications) in lithium ion batteries, supercapacitors, thermoelectrics, and in bone tissue scaffolds $[1,5-8]$.

Carbon fibers are also sought for shielding of electromagnetic (EMI) interference $[9,10]$. EMI is a phenomenon that causes the degradation/interception in the performance of electronic equipment. The need to overcome EMI has rapidly grown with the increased development of portable electronic and wireless communication devices. Carbon nanomaterials such as vapor grown carbon nanofibers and CNTs have shown potential as reinforcing agents in polymers (which have transparency to EMI). Given the high specific surface area and electrical conductivity, these type of nanofillers show improved EMI shielding [11]. Carbon nanomaterials have shown high surface area, for example, Bi et al. have reported a value of $423 \mathrm{~m}^{2} / \mathrm{g}$ in surface area for graphene[12] and Hashim et al. reported $360 \mathrm{~m}^{2} / \mathrm{g}$ for CNTs [6]. Reports have shown SE values that range from 7 to $45 \mathrm{~dB}$ for nanoreinforced polymer composites [10,13-15]. According to Yang et al. a shielding 
effectiveness of $30 \mathrm{~dB}$ would satisfy $50 \%$ of the applications in the automotive and electronic/computer industries, while $40 \mathrm{~dB}$ would satisfy $95 \%$ of industry requirements [10].

Polyacrylonitrile (PAN) has traditionally been the precursor of choice for regular (micro/macro) high-performance carbon fibers though the high cost associated with the lengthy and energy intensive oxidation stabilization steps and high carbonization temperature, has hindered its wide acceptance as the material of choice for many applications. The cost of the raw material is high compared to the post-processing steps (stabilization, sizing, surface treatment, etc.). The usage of PAN based carbon fibers have therefore been limited to high performance structural applications. At the nanoscale, carbon fibers derived from polymer precursors have traditionally been produced mainly through electrospinning processes followed by oxidation/carbonization/graphitization methods as used for PAN. These fibers may have diameters ranging from hundreds of nanometers to several microns depending on the selected spinning parameters used to manufacture the polymeric fiber precursors [16-18]. The industrial potential for carbon fibers prepared through this method is slim given the high cost of the polymer precursor (PAN), the low yield of the electrospinning process, and the lengthy and high cost associated with carbonization steps.

Water soluble polymers such as polyvinyl alcohol (PVA) and polyvinyl butyral (PVB) have shown potential as low cost precursors for carbon fiber production. These polymers have low toxicity, cost effectiveness, and sufficient carbon content $[19,20]$. Recently, Cremar et. al. published on the development of carbon fine fibers utilizing PVA as the polymer precursor with subsequent exposure to sulfuric acid vapor followed by a low temperature heat treatment. Its initial characterization shows that the developed fibers, that were exposed to sulfuric acid vapors, possess a graphitic carbon structure as observed in a Raman spectroscopy study (crystalized graphite peak at $1580 \mathrm{~cm}^{-1}$ ) [21]. Further heat treatment heightened the graphitic formation and resulted in an increased thermal stability 
with no apparent degradation below $800^{\circ} \mathrm{C}$. These carbon fibers show homogeneous, continuous, and structurally intact fiber morphology with an average diameter of $290 \mathrm{~nm}$ for the PVA precursor fibers and $191 \mathrm{~nm}$ for the carbonized fibers. The density of these carbon fiber mats ( $1 \times 1 \mathrm{~cm}$ nonwoven mat with $0.06 \mathrm{~mm}$ thickness) was shown to be $\sim 165 \mathrm{mg} / \mathrm{cm}^{3}$ while the Brunauer-Emmett-Teller (BET) specific surface area, $\mathrm{S}_{B E T}$ was reported to be 576 $\mathrm{m}^{2} \mathrm{~g}^{-1}$, and the multipoint BET analysis indicated that the $\mathrm{N}_{2}$ adsorption isotherm of the carbon nanofiber mat has micro and macro-porous attributes with an average pore size of 100 $\mathrm{nm}$ [21]. In this paper we report on the electrical resistivity, shielding of electromagnetic interference, and mechanical properties of the developed carbon fiber mats.

\section{Results and Discussion}

\subsection{Electrical measurements}

Solutions of PVA and deionized water were prepared with concentrations ranging from $5-14 \%$ (wt./wt.) of PVA. It was observed that as the polymer concentration increased, the number of beads along the fibers decreased, however, this also resulted in an increased fiber diameter. Optimization studies were conducted with the goal of obtaining small fiber diameter with low to negligible number of beads. A concentration of $10 \%$ (wt./wt.) was chosen as the optimum concentration and nonwoven PVA nanofiber mats were developed with varying grams per square meter (GSM). The varying GSM mats provided venues to analyze the influence of the carbonization process and resultant electrical and mechanical properties. Fig. 1 shows pictures of the resultant PVA fibers precursor mat (a), exposure of the mat to sulfuric acid vapor (b) and carbonized mat (c). Each of these mats is shown with their respective scanning electron micrographs (d, e, and f). 
a

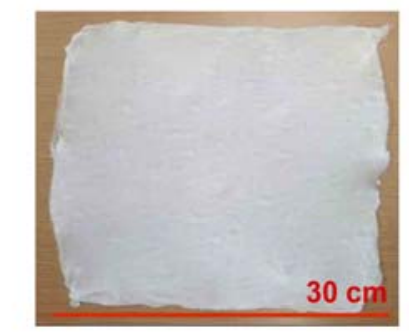

b

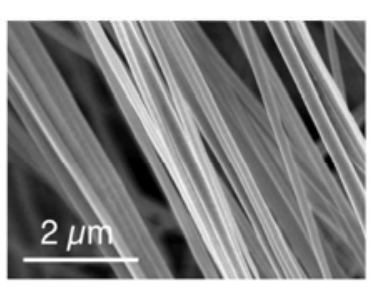

C

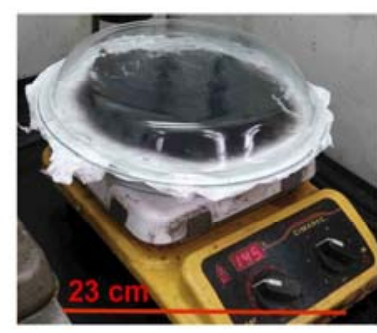

d

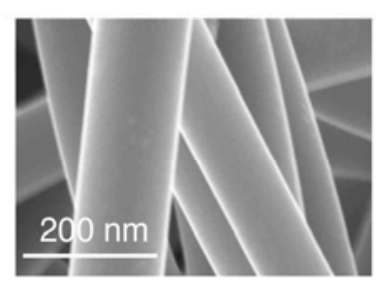

e
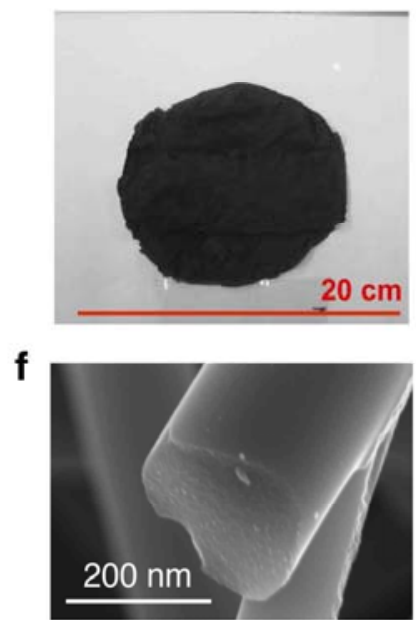

Fig. 1. Digital pictures of the produced carbon fiber mats with their corresponding scanning electron micrographs. (a \& b) Nonwoven PVA fiber mats; (c \& d) PVA fibers mats subjected to a sulfuric acid vapor dehydration process in air; and (e \& f) nonwoven dehydrated mats subjected to a low temperature heat-treatment process to obtain high performance multi-functional carbon fibers.

Surface and volume resistivity measurements were taken for the PVA, acid vapor dehydrated, and heat-treated fiber mats (Fig. 2). Electrical resistivity was obtained utilizing equations 1 and 2, where $(\rho)$ is volume resistivity, (A) is the cross-sectional area and $(I)$ is the thickness of the mats. The electrical volume resistivity of the pure PVA mats was shown to be $8 \times 10^{14} \Omega \cdot \mathrm{cm}$ (Fig. 2c) [22]. A drop of twelve orders of magnitude was observed for the acid treated mats showing a resistivity of $165 \Omega \cdot \mathrm{cm}$ (conductivity of $6.1 \times 10^{-3} \mathrm{~S} \cdot \mathrm{cm}^{-1}$ ), while the volume resistivity further decreased upon heat treatment to $0.407 \Omega \cdot \mathrm{cm}$ (conductivity of $\left.2.46 \mathrm{~S} \cdot \mathrm{cm}^{-1}\right)$. The decreased resistivity is due to the formation of graphitic structures ( $\mathrm{G}$ band at $1580 \mathrm{~cm}^{-1}$ corresponding to the in-plane stretching $E_{2 \mathrm{~g}}$ vibration mode of crystallized graphite )[21], and was found to be slightly less than that of the CNT sponges developed by Hashim et al. [6]. The surface resistivity $\left(\rho_{\mathrm{s}}\right)$ of the partially carbonized (only exposed to sulfuric acid vapors) and heat treated (heat treated after exposure to sulfuric acid vapors) mats was also measured with a four-point probe apparatus and calculated with equation 2 where $(V)$ is the measured voltage and $(I)$ is the current that passes through the probes onto the 
mat. The sheet resistivity of both the acid and acid-heat treated fiber mats was found to be 18 $\mathrm{k} \Omega / \mathrm{sq}$ and $59 \Omega / \mathrm{sq}$ respectively. The measured sheet resistivities for these carbon fibers are similar to those of nanoporous carbon fibers ( 35 to $56 \Omega /$ sq) synthesized via template chemical vapor deposition [23]. In here, it should be noted that the surface resistivity value obtained from four point probe measurements is not considered a highly accurate value, since the fibers show isotropic orientation and high porosity which interferes with the location of the probes. This is the reason why the resistivity is observed to be higher than the values obtained from volume resistivity measurements.

$$
\begin{array}{r}
\rho=R \frac{A}{l} \\
\rho_{S}=\left(\frac{\pi}{\ln (2)}\right) \frac{V}{I}
\end{array}
$$



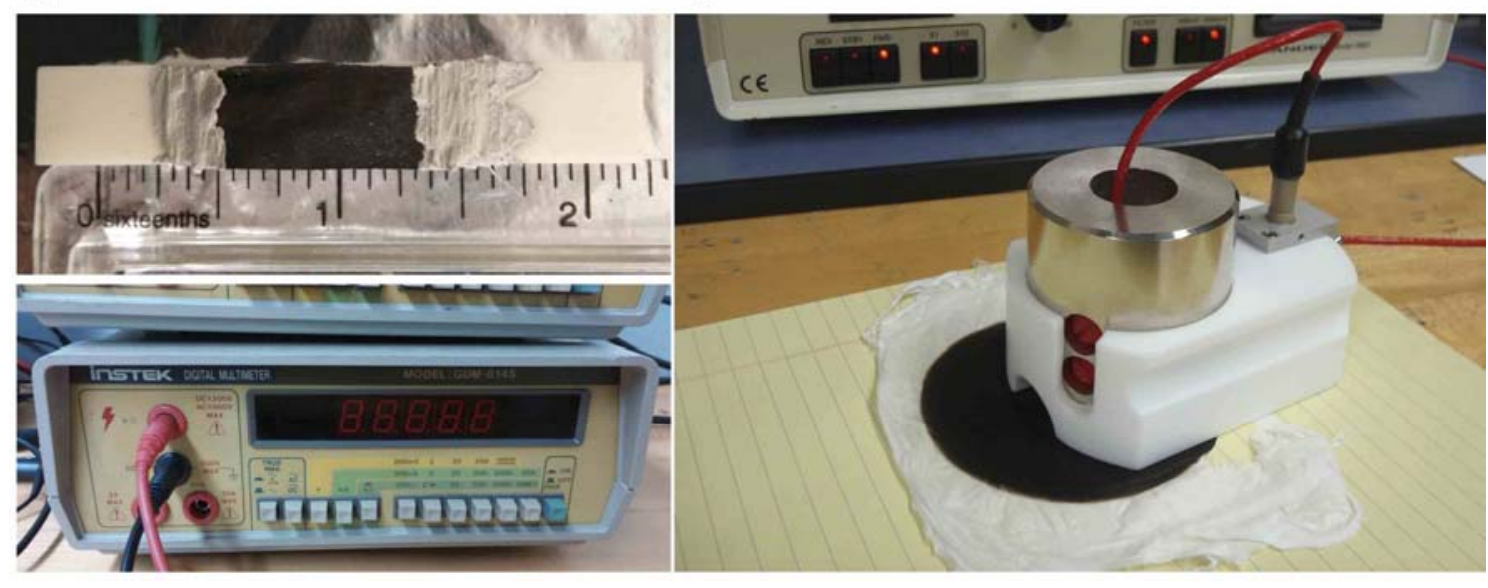

C

Electrical measurements

\begin{tabular}{|c|c|c|c|}
\hline & $\begin{array}{c}\text { Volume resistivity } \\
(\Omega \cdot \mathrm{cm})\end{array}$ & $\begin{array}{c}\text { Conductivity } \\
\left(\mathrm{S} \cdot \mathrm{cm}^{-1}\right)\end{array}$ & $\begin{array}{c}\text { Sheet resistivity } \\
(\Omega / \mathrm{sq})\end{array}$ \\
\hline PVA & $8 \times 10^{14}$ & $1.3 \times 10^{15}$ & $1.8 \times 10^{4}$ \\
\hline Acid-treated PVA & 165 & $6.1 \times 10^{-3}$ & 59 \\
\hline Carbon nanofiber & 0.407 & 2.46 & 5 \\
\hline
\end{tabular}

Fig. 2. Electrical resistivity measurements. (a) A heat-treated carbon fiber mat is placed on a ceramic surface, and colloidal silver paste (Ted Pella, Inc Product No. 16032) is placed at both ends of the sample. A digital multimeter (instek model GDM-8145) is used to measure the resistance value $(R)$. The volume resistivity is then measured by measuring the cross sectional area of the mat $(A)$ and thickness of the sample (l). (b) Four point probe measurements were taken with the Jandel Model RM2 to calculate the sheet resistivity (equation 2) where $I$ is the current (amps) and $V$ is the measured voltage across the sample. (c) Electrical measurements for PVA, acid-treated PVA, and carbon nanofiber mats.

\subsection{Electromagnetic Interference Shielding Effectiveness}

The electromagnetic interference shielding effectiveness (attenuation upon

transmission) was analyzed using a fixture constructed based on the transmission line theory

[24]. The setup consisted of a sample holder with input and output connected to the network

analyzer as shown in Fig. 3. Sample geometries and schematics of the holder are described

elsewhere [10]. The holder is connected to a coaxial transmission line, and two sample

geometries, referred as reference and load, are utilized. Shielding effectiveness (SE) is

obtained from the difference between the load and reference sample. 


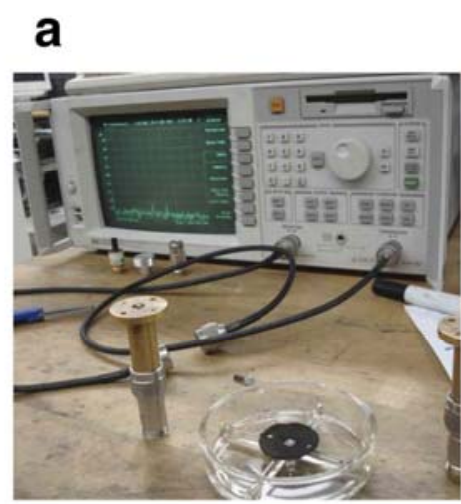

b

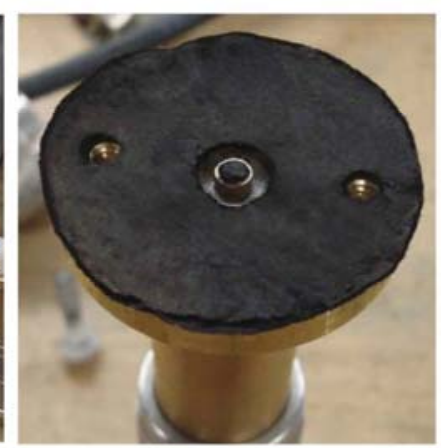

C

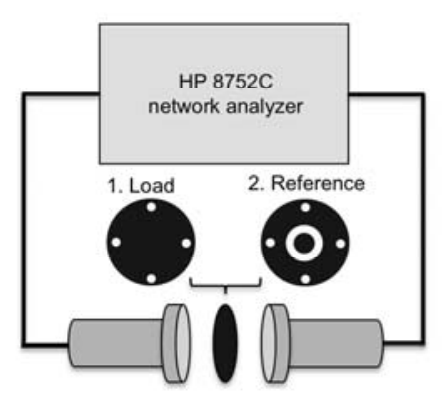

Fig. 3. The shielding efficiency of nonwoven carbon fiber mats was measured utilizing a coaxial flanged fixture connected to a Hewlett Packard 8752C network analyzer (a). Two carbon nanofiber sample geometries are required (load and reference) (b) and each was measured separately by placing it between the coaxial flanged fixtures, pictures shows reference sample (c).

Measurements were conducted at frequency range of 0 to $1.5 \mathrm{GHz}$ and results are shown in Fig. 4. The EMI SE of pure PVA was measured and as expected the sample is transparent to electromagnetic radiation, with an SE of zero. The vapor treated PVA nonwoven fiber mat showed an SE value of $30 \mathrm{~dB}$ at $300 \mathrm{MHz}$ and $20 \mathrm{~dB}$ at $1.3 \mathrm{GHz}$. As expected, reflection and absorption mechanisms are enhanced with the rise in electrical conductivity. In the case of the fully carbonized heat-treated mats, the SE was observed to increase to $45 \mathrm{~dB}$ at $300 \mathrm{MHz}$ and $40 \mathrm{~dB}$ at $1.3 \mathrm{GHz}$. 

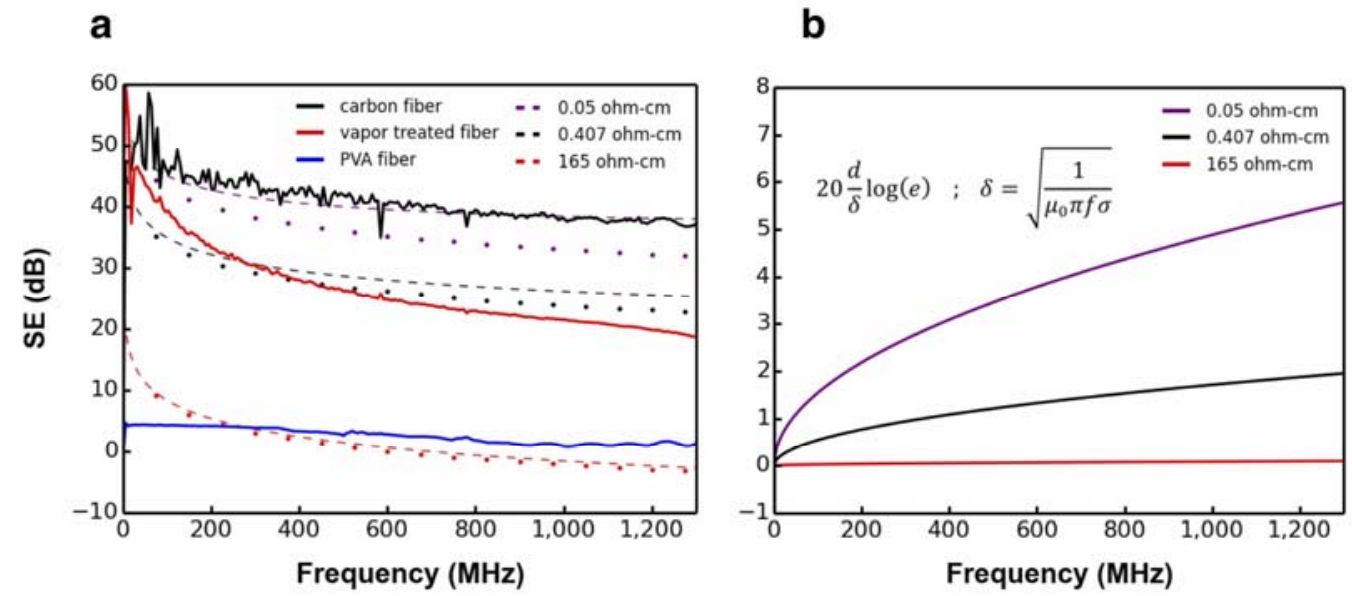

Fig. 4. The shielding efficiency for the nonwoven PVA, vapor treated, and carbon fiber mat are shown in Fig 4(a). Also shown is the predicted shielding efficiency (SE) using the Simon (dotted) and Colaneri (dashed) formalism for (a) the nonwoven vapor treated (red, $165 \mathrm{ohm}$ $\mathrm{cm}$ ), carbon fiber (black, $0.407 \mathrm{ohm}-\mathrm{cm}$ ), and a sample with a conductivity of $0.05 \mathrm{ohm}-\mathrm{cm}$ (purple). The sample thickness is approximately $0.2 \mathrm{~mm}$. The predicted shielding effect using the Colaneri formalism is stabilized as the conductivity and frequency increase, which is attributed to the absorption term shown in (b). The skin depth decreases over the frequency range, which results in an increase of the absorption term and is most notable for good conductors.

EMI shielding results from reflection, absorption, and multiple reflections

mechanisms, though commonly expressed according to the Simon formalism where multiple reflections are ignored (equation 3). The obtained theoretical SE value is based on frequency and electrical conductivity values [10].

$$
S E=50+10 \log _{10}(\rho f)^{-1}+1.7 t\left(\frac{f}{\rho}\right)^{1 / 2}
$$

Where SE is in decibels $(\mathrm{dB}), \rho$ is the volume resistivity $(\Omega \cdot \mathrm{cm})$ at room temperature, $t$ is the thickness of the sample $(\mathrm{cm})$, and $f$ is frequency $(\mathrm{MHz})$. According to the Simon formalism, the SE of the measured samples would have been $3(-3) \mathrm{dB}$ and $30(25) \mathrm{dB}$ for the acid treated and heat treated mats at $300 \mathrm{MHz}(1300 \mathrm{MHz})$. As mentioned, multiple reflections are ignored given the predominance of reflection and absorption mechanisms for traditional solid samples. The higher SE values obtained from the experimental results are attributed to the contribution from multiple reflections. As previously reported, porous materials such as nonwoven nanofiber mats have high specific surface area, $576 \mathrm{~m}^{2} \mathrm{~g}^{-1}$ [13], and high interfacial 
ratios, which effectively contribute to the multiple reflection mechanisms and increase the value of SE [21].

At high frequencies, the stability of the SE varies for the chemically treated and subsequently heat treated mats. For the latter samples the drop is not as noticeable as for the chemically treated fibers. This result is attributed to skin effects. According to Colaneri et al. [25], at high frequencies the absorption term can dominate. Following the 'good conductor' approximation $\left(\sigma / 2 \pi f \epsilon_{0}=7.6\right.$ for the vapor treated at $\left.1.5 \mathrm{GHz}\right)$ in the far field, when the thickness of the material exceeds the skin depth, the shielding effect can be expressed as:

$$
S E=10 \log \left(\frac{\sigma}{32 \pi f \epsilon_{0}}\right)+20 \frac{d}{\delta} \log (e) \quad ; \delta=\sqrt{\frac{1}{\mu_{0} \pi f \sigma}}
$$

Where sigma is the bulk conductivity $(\mathrm{S} / \mathrm{cm}), f$ is the frequency in Hertz, $\epsilon_{0}$ is the permittivity of free space $8.854 \times 10^{-14}(\mathrm{~F} / \mathrm{cm}), d$ is the thickness $(\mathrm{cm}), \delta$ is the skin depth, and the permeability is $\mu_{0}=4 \pi \times 10^{-9} \mathrm{H} / \mathrm{cm}$. The first term on the right hand side is associated with single reflections and the second is attenuation by absorption. The skin effect results in electromagnetic radiation penetrating at the near surface only at high frequencies. For conductive materials, as the frequency increases, the skin depth becomes smaller resulting in an increase of the absorption term and overall shielding effectiveness. Fig. 4a, shows the predicted SE according to the Colaneri and Simon formalism using resistivity values for the vapor treated and heat treated carbon fibers, along with a lower resistivity value of $0.05 \Omega \cdot \mathrm{cm}$ just to exemplify the effect of conductivity on the stability of the SE with increasing frequency. Although the reflection term contributes greatly to the SE, this can change as the frequency increases where the increased stability of the SE is associated with decreased skin depth seen in the absorption term (Fig. 4b). Chung, et. al. [14] showed that carbon-carbon composites with continuous carbon fibers can have severe skin effects with abrupt increase in surface impedance at large frequencies. The SE of the carbon-carbon composite remained steady up to a frequency range of $1.5 \mathrm{GHz}$. Carbon fiber has also found use as microwave 
absorbing materials $(8-12 \mathrm{GHz})$ for use in the military and commodity markets. Composites filled with increasing amounts of carbon nanofibers or porous nanofibers have been shown to improve the absorption at frequencies ranging up to $12 \mathrm{GHz}$. In these composites, it is believed that the mechanism for microwave enhanced absorption, with increasing carbon fiber amount, is due to a dielectric-loss of electromagnetic waves or dielectric dispersion properties [26,27].

\subsection{Mechanical properties}

The mechanical properties of the developed carbon fiber mats was evaluated by dynamic mechanical analysis (Fig. 5). For this test, PVA fibers were collected in a unidirectional fashion and twisted along the fiber axis to prepare fiber yarns. Acid vapor treatment and heat treatment of the fibers was carried out in the same manner with slight modifications to the reaction apparatus. All yarns were subsequently dipped in a $10 \%$ (wt./wt.) PVA solution to improve handling of the yarns [28]. The modulus of elasticity for the PVA NFs, acid treated NF mats, and heat-treated NF mats was found to be $0.5,0.5$, and 3.0 GPa, respectively. A tensile strength of $124 \mathrm{MPa}$ with $21 \%$ strain and a toughness of 1.8 $\mathrm{kJ} \mathrm{m}^{-3}$ were obtained for the heat-treated nonwoven fiber mats. These values are similar to those reported for buckypaper and graphene-PVA composites[29,30]. 


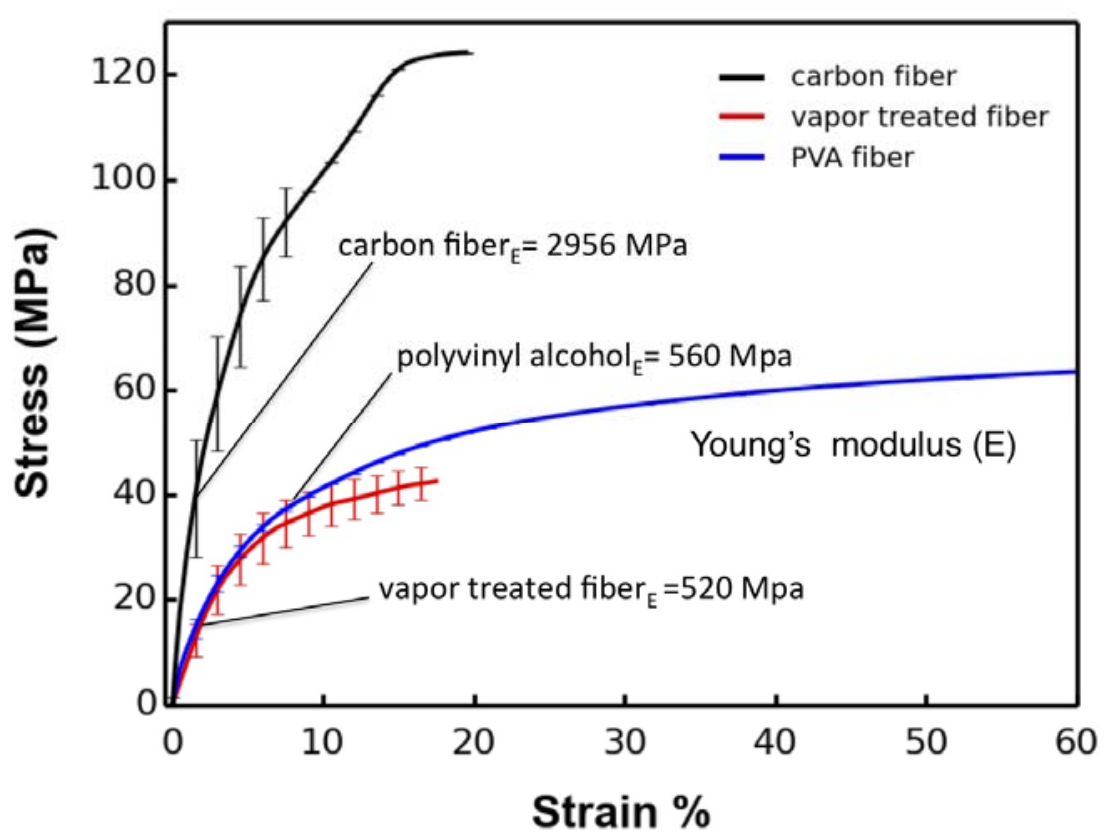

Fig. 5. Stress-strain curve produced through dynamic mechanical analysis of the developed carbon fibers. The toughness (area under the stress-strain curve) was found to be $1876 \mathrm{~J} / \mathrm{m}^{3}$ for the heat treated carbon fibers, $538 \mathrm{~J} / \mathrm{m}^{3}$ for the vapor treated, and $4407 \mathrm{~J} / \mathrm{m}^{3}$ for the PVA precursor fibers respectively.

\section{Experimental}

\subsection{Materials}

Polyvinyl alcohol (Average Mw 85-124K, 96\% hydrolyzed) and 98\% sulfuric acid were purchased from Sigma Aldrich. The lab scale Cyclone ${ }^{\mathrm{TM}} \mathrm{L}-1000 \mathrm{M}$ from Fiberio Technology Corp. was used for production of fine fibers.

\subsection{General methods}

The nonwoven carbon fiber mats are prepared as follows: A 10\% (wt./wt.) solution of polyvinyl alcohol (PVA) is prepared by adding $1.11 \mathrm{~g}$ of PVA (Average MW 85-124K, 96\% hydrolyzed from Sigma Aldrich) to $10 \mathrm{~mL}$ of deionized water. The components are stirred with a magnetic bar in an oil bath at $75^{\circ} \mathrm{C}$ until clear. The lab scale Cyclone ${ }^{\mathrm{TM}} \mathrm{L}-1000 \mathrm{M}$ from FibeRio Technology Corp. was used for the production of the fine fibers. The nonwoven mat is prepared as follows: $1 \mathrm{ml}$ of the obtained PVA aqueous solution is injected into the Cyclone spinneret and spun at 7000 RPM for 1 minute. The produced fibers are 
allowed to collect on a flat substrate placed on a vacuum box. The procedure is repeated until the desired grams per square meter (GSM) are obtained. The PVA fiber mats are then exposed to sulfuric acid vapors at temperatures ranging from room temperature up to 200

${ }^{\circ} \mathrm{C}$. Following chemical treatment, the mats are heat treated in a tube furnace. The nonwoven sulfuric acid vapor treated PVA fiber mat is washed with water and heated, with a ramping rate of $5{ }^{\circ} \mathrm{C} \mathrm{min}^{-1}$, to $800{ }^{\circ} \mathrm{C}$ and held for 20 minutes. All samples are heat treated under an inert atmosphere (Nitrogen gas).

\subsection{Characterization}

The morphology of the fiber mats was extensively studied by scanning electron microscopy (SEM, Sigma VP, Carl Zeiss, Germany). The electrical conductivity was analyzed using a four-point probe tester JANDEL RM2 (Jandel Engineering, Ltd., USA). The instek model GDM-8145 was used to measure the resistance of the samples. The shielding effectiveness of the electromagnetic interference was conducted using an in house fixture based on the coaxial transmission line theory and connected to a $8712 \mathrm{C}$ RF Network Analyzer (Hewlett Packard) [24]. Mechanical properties were analyzed utilizing a dynamic mechanical analyzer TA-Q series model Q800. The stress-strain runs had a sample size of 5 $\mathrm{mm}$ in length and $0.2 \mathrm{~mm}$ in diameter with a ramping rate of $0.001 \mathrm{~N} / \mathrm{min}$. All carbon and carbon composite samples were dipped in a 10\% (wt./wt.) PVA aqueous solution and allowed to dry before initiating mechanical tests.

\section{Conclusion}

This study presents the analysis of the mechanical and electrical properties of carbon nonwoven nanofibers mats produced from a water-soluble polymer precursor using the Forcespinning ${ }^{\circledR}$ technology. The nonwoven nanofiber mats show high shielding effectiveness of EMI and mechanical integrity making it a material with promising potential applications not only in filtration and as oil spill sorbents, but attractive in the electrical and electronic 
industries for energy storage, and development of light-weight high voltage cables. The observed SE indicates that these carbon nonwoven mats have use in electrical components found in aerospace vehicles where shielding and light weight are of upmost importance. Because of the increased need of electrical components in airplanes, the production of the nonwoven carbon mats must be simple and effective for low-cost production, scalability, and reproducibility needed for industrialization of carbon-based materials. The presented dehydration induced process coupled with low temperature carbonization presents an attractive method to develop multifunctional carbon nonwoven mats that could be used "as is" or embedded in polymer composites for reinforcement.

\section{Acknowledgements}

The authors gratefully acknowledge support received from National Science Foundation under PREM grant No. DMR-1523577 “UTRGV-UMN Partnership for Fostering Innovation by Bridging Excellence in Research and Student Success". 


\section{References}

[1] Y. A. Kim, T. Hayashi, M. Endo, M. S. Dresselhaus, Carbon Nanofibers. In Springer

Handbook of Nanomaterials, Vajtai, R. E., (ed.) Springer-Verlag Berlin Heidelberg(2013), pp 233

[2] N. Behabtu, C. C. Young, D. E. Tsentalovich, O. Kleinerman, X. Wang, A. W. K. Ma, et al., Fibers of carbon nanotubes with ultrahigh conductivity, Science 339 (2013) 182-186.

[3] A. B. Dalton, S. Collins, E. Munoz, J. M. Razal, V. H. Ebron, J. P. Ferraris, et al., Super-tough carbon-nanotube fibres, Nature 423 (2003) 703-703.

[4] M. E. Kozlov, R. C. Capps, W. M. Sampson, V. H. Ebron, J. P. Ferraris, R. H. Baughman, Spinning solid and hollow polymer-free carbon nanotube fibers, Adv. Mater. 17 (2005) 614-617.

[5] C. A. Hewitt, A. B. Kaiser, S. Roth, M. Craps, R. Czerw, D. L. Carroll, Multilayered Carbon Nanotube/Polymer Composite Based Thermoelectric Fabrics, Nano Lett. 12 (2012) 1307-1310.

[6] D. P. Hashim, N. T. Narayanan, J. M. Romo-Herrera, D. A. Cullen, M. G. Hahm, P. Lezzi, et al., Covalently bonded three-dimensional carbon nanotube solids via boron induced nanojunctions, Sci. Rep. 2 (2012) 1-8.

[7] W. Dai, S. J. Kim, W.-K. Seong, S. H. Kim, K.-R. Lee, H.-Y. Kim, et al., Porous carbon nanoparticle networks with tunable absorbability, Sci. Rep. 3 (2013) 1-8.

[8] J. Lee, J. Kim, T. Hyeon, Recent progress in the synthesis of porous carbon materials, Adv. Mater. 18 (2006) 2073-2094.

[9] D. D. L. Chung, Electromagnetic interference shielding effectiveness of carbon materials, Carbon 39 (2001) 279-285.

[10] S. Yang, K. Lozano, A. Lomeli, H. D. Foltz, R. Jones, Electromagnetic interference shielding effectiveness of carbon nanofiber/LCP composites, Compos. Part A-Appl. S. 36 (2005) 691-697.

[11] X. Fu, and D. D. L. Chung, Submicron carbon filament cement-matrix composites for electromagnetic interference shielding, Cem. Concr. Res. 26 (1996) 1467-1472.

[12] H. Bi, X. Xie, K. Yin, Y. Zhou, S. Wan, L. He, et al., Spongy graphene as a highly efficient and recyclable sorbent for oils and organic solvents, Adv. Funct. Mater. 22 (2012) 4421-4425.

[13] M. H. Al-Saleh, and U. Sundararaj, A review of vapor grown carbon nanofiber/polymer conductive composites, Carbon 47 (2009) 2-22.

[14] X. Luo, and D. D. L. Chung, Electromagnetic interference shielding using continuous carbon-fiber carbon-matrix and polymer-matrix composites, Compos. Part B-Eng. 30 (1999) 227-231.

[15] B. Weng, F. Xu, A. Salinas, K. Lozano, Mass production of carbon nanotube reinforced poly(methyl methacrylate) nonwoven nanofiber mats, Carbon 75 (2014) 217-226.

[16] S. N. Arshad, M. Naraghi, I. Chasiotis, Strong carbon nanofibers from electrospun polyacrylonitrile, Carbon 49 (2011) 1710-1719.

[17] M. A. Hunt, T. Saito, R. H. Brown, A. S. Kumbhar, A. K. Naskar, Patterned functional carbon fibers from polyethylene., Adv. Mater. 24 (2012) 2386-9.

[18] J. M. Younker, T. Saito, M. A. Hunt, A. K. Naskar, A. Beste, Pyrolysis pathways of sulfonated polyethylene, an alternative carbon fiber precursor., J. Am. Chem. Soc. 135 (2013) 6130-41.

[19] J. Spender, A. L. Demers, X. Xie, A. E. Cline, M. A. Earle, L. D. Ellis, et al., Method for production of polymer and carbon nanofibers from water-soluble polymers., Nano Lett. 12 (2012) 3857-60. 
[20] M. Inagaki, Y. Yang, F. Kang, Carbon nanofibers prepared via electrospinning., Adv. Mater. 24 (2012) 2547-66.

[21] L. D. Cremar, J. Acosta-Martinez, A. Villarreal, A. Salinas, L. Wei, Y. Mao, et al., High-Throughput of Multifunctional Carbon Nanofibers from Water Soluble Precursors, Chem. Fibers Int. CFI-1 (2016) 40-42.

[22] M. F. M. A. Zamri, S. H. S. Zein, A. Z. Abdullah, N. I. Basir, Improved electrical conductivity of polyvinyl alcohol/ multiwalled carbon nanotube nanofibre composite films with $\mathrm{MnO} 2$ as filler synthesised using the electrospinning process., IJET 11 (2011) 20-25.

[23] K. Sawada, S. Sakai, M. Taya, Fabrication of ultrafine carbon fibers possessing a nanoporous structure from electrospun polyvinyl alcohol fibers containing silica nanoparticles, J. Nano Mat. 2014 (2014) 6.

[24] H. Vasquez, L. Espinoza, K. Lozano, H. D. Foltz, S. Yang, Simple Device for Electromagnetic Interference Shielding Effectiveness Measurement, IEEE (2009) 62-68.

[25] N. F. Colaneri, and L. W. Schacklette, EMI shielding measurements of conductive polymer blends, IEEE Trans. Instr. Meas. 41 (1992) 291-297.

[26] G. Li, T. Xie, S. Yang, J. Jin, J. Jiang, Microwave Absorption Enhancement of Porous Carbon Fibers Compared with Carbon Nanofibers, J. Phys. Chem. C 116 (2012) 9196-9201.

[27] N. Tang, W. Zhong, C. Au, Y. Yang, M. Han, K. Lin, et al., Synthesis, Microwave Electromagnetic, and Microwave Absorption Properties of Twin Carbon Nanocoils, J. Phys. Chem. C 112 (2008) 19316-19323.

[28] A. M. Beese, S. Sarkar, A. Nair, M. Naraghi, Z. An, A. Moravsky, et al., Bio-inspired carbon nanotube-polymer composite yarns with hydrogen bond-mediated lateral interactions, ACS Nano 7 (2013) 3434-46.

[29] A. Aldalbahi, and M. in het Panhuis, Electrical and mechanical characteristics of buckypapers and evaporative cast films prepared using single and multi-walled carbon nanotubes and the biopolymer carrageenan, Carbon 50 (2012) 1197-1208.

[30] M. Cano, U. Khan, T. Sainsbury, A. O’Neill, Z. Wang, I. T. McGovern, et al., Improving the mechanical properties of graphene oxide based materials by covalent attachment of polymer chains, Carbon 52 (2013) 363-371. 